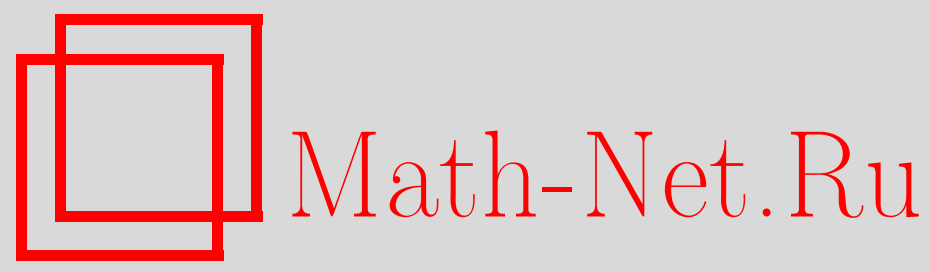

М. В. Балашов, О геометрической разности многозначных отображений, Матем. заметки, 2001, том 70, выпуск 2, 163-169

DOI: https://doi.org/10.4213/mzm730

Использование Общероссийского математического портала Math-Net.Ru подразумевает, что вы прочитали и согласны с пользовательским соглашением http://www.mathnet.ru/rus/agreement

Параметры загрузки:

IP : 3.82 .47 .9

26 апреля 2023 г., $15: 24: 17$

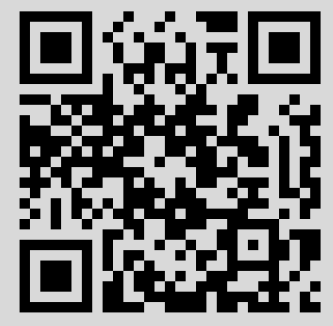




\section{О ГЕОМЕТРИЧЕСКОЙ РАЗНОСТИ МНОГОЗНАЧНЫХ ОТОБРАЖЕНИЙ}

\section{М.В. Балашов}

Получен аналог конечномерной теоремы о полунепрерьвности сверху геометрической разности непрерывных многозначных отображений для сепарабельных банаховых пространств. Получены достаточные условия непрерывности геометрической разности многозначных отображений в конечномерном пространстве без предположения об условии "непустой внутренности". Приведены примеры, показывающие неулучшаемость полученных результатов.

Библиографояи: 5 названий.

1. Введение. Геометрическая разность является важной теоретико-множественной операцией, имеющей большое значение в выпуклом анализе и в линейных дифференциальных играх.

Наиболее существенным для приложений является вопрос о сохранении непрерывности (или полунепрерывности) геометрической разности непрерывных (полунепрерывных) многозначных отображений. Этим вопросам посвящена данная статья.

Пусть $\mathscr{T}$ - метрическое пространство, $\mathscr{Y}$ - векторное полное метрическое пространство с инвариантной относительно сдвигов метрикой.

ОПРЕДЕЛЕНИЕ. Геометрической разностью множеств $A$ и $B$ из векторного пространства У назьвается множество

$$
A \stackrel{*}{*}=\{x \mid x+B \subset A\} .
$$

Напомним определения полунепрерывности многозначных отображений (см. определения 1,2 гл. $3, \S 1$ из [1], а также [2]).

Многозначное отображение $F: \mathscr{T} \rightarrow \mathscr{Y}$ назьвается полунепрерывным сверху (пн. cв.) $\quad$ в точке $t_{0} \in \mathscr{T}$, если для любой окрестности $N\left(F\left(t_{0}\right)\right)$ множества $F\left(t_{0}\right)$ сушествует окрестность $U\left(t_{0}\right)$ точки $t_{0}$ такая, что для всех $t \in U\left(t_{0}\right)$ имеет место вложение $F(t) \subset N\left(F\left(t_{0}\right)\right)$.

Многозначное отображение $F: \mathscr{T} \rightarrow \mathscr{Y}$ назьвается полунепрерывным снизу (пн. cн.) $\boldsymbol{в}$ точке $t_{0} \in \mathscr{T}$, если для любой точки $y_{0} \in F\left(t_{0}\right)$ и для любой окрестности $N\left(y_{0}\right)$ точки $y_{0}$ существует окрестность $U\left(t_{0}\right)$ точки $t_{0}$ такая, что для всех $t \in U\left(t_{0}\right)$ $F(t) \cap N\left(y_{0}\right) \neq \varnothing$.

Работа выполнена при поддержке Российского фонда фундаментальных исследований (грант 98-01-00645). 
Будем говорить, что многозначное отображение $n н . \quad$ св. (соответственно сн.) в слабой топологии пространства У (или просто слабо пн.), если определения пн. св. (сн.) выполнены для любой слабой окрестности $N\left(F\left(t_{0}\right)\right)$ (соответственно $\left.N\left(y_{0}\right)\right)$.

Пусть $B_{\varepsilon}(0)=\{x \in \mathscr{Y} \mid \varrho(0, x) \leqslant \varepsilon\}-\varepsilon$-шар метрического пространства У. Мы будем пользоваться также определением $\varepsilon$-пн. св. и пн. сн.; именно, будем говорить, что отображение $F: \mathscr{T} \rightarrow \mathscr{Y} \varepsilon-n н$. св. в точке $t_{0}$, если

$$
\forall \varepsilon>0 \exists U\left(t_{0}\right) \forall t \in U\left(t_{0}\right) \quad F(t) \subset F\left(t_{0}\right)+B_{\varepsilon}(0) ;
$$

отображение $F: \mathscr{T} \rightarrow \mathscr{Y} \varepsilon$-пн. сн. в точке $t_{0}$, если

$$
\forall \varepsilon>0 \exists U\left(t_{0}\right) \forall t \in U\left(t_{0}\right) \quad F\left(t_{0}\right) \subset F(t)+B_{\varepsilon}(0) .
$$

Под непрерывностью $F$ в точке $t_{0}$ мы будем понимать пн. св. и пн. сн. в точке $t_{0}$.

Под непрерывностью в метрике Хаусдорфа мы будем понимать $\varepsilon$-пн. св. и сн.

Приведем известные случаи, когда геометрическая разность (полу)непрерьвна.

УТВЕРЖДЕНИЕ $1.1\left[2, \S 4\right.$, теорема 3 , пример 4]. Пусть $F, G: \mathscr{T} \rightarrow \mathbb{R}^{n}$ - многознач-

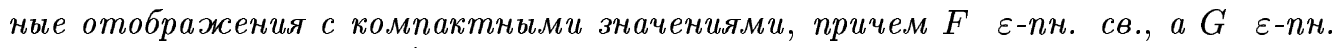
сн. на $\mathscr{T} u H(t)=F(t) \stackrel{*}{G}(t) \neq \varnothing$ для всех $t$. Тогда отображсение $H(t)$ пн. св. Из непрерывности $F$ и $G$ не следует непрерывность $H$.

УТВЕРЖДЕНИЕ 1.2 [3], [2, §4, следствие 3 теоремы 3 , теорема 7]. Пусть $F, G: \mathscr{T} \rightarrow \mathbb{R}^{n}$ - многозначные отображения с компактными значениями, непрерывные в точке $t_{0} \in \mathscr{T}, u F(t)$ имеет выпуклье значения для всех $t$. Пусть $\operatorname{int}\left(F\left(t_{0}\right) \stackrel{*}{*} G\left(t_{0}\right)\right) \neq \varnothing$ (т.н. условие "непустой внутренности"). Тогда $F(t) \stackrel{*}{*} G(t)$ непрерывно в точке $t_{0}$.

При сохранении условия “непустоты внутренности" и звездности множест-

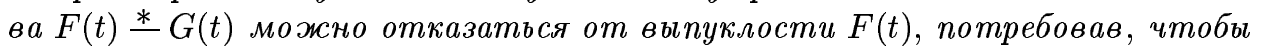

$$
\exists w>0 \forall t \exists v_{0}>w \forall v \in\left(0, v_{0}\right) \quad\left(F(t)+B_{v}(0)\right) \stackrel{*}{-} B_{v}(0)=F(t) .
$$

В работе рассматриваются новые свойства непрерывности операции геометрической разности многозначных отображений с ограниченными значениями в сепарабельных банаховых пространствах и в $\mathbb{R}^{n}$.

В случае сепарабельного банахова пространства, снабженного слабой топологией, показано, что геометрическая разность двух многозначных отображений при определенных условиях полунепрерывна сверху.

В конечномерном случае получены достаточные условия, при которых геометрическая разность двух многозначных отображений непрерывна без условия "непустой внутренности".

2. Полунепрерывность сверху разности многозначных отображений в банаховых пространствах. Отметим, что для многозначного отображения с компактными значениями понятия пн. и $\varepsilon$-пн. в метрическом пространстве совпадают.

ЛЕмма 2.1. Пусть $F: \mathscr{T} \rightarrow \mathscr{Y}$ - многозначное отображение.

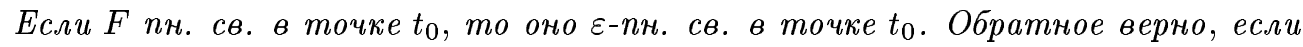
$F\left(t_{0}\right)$ - компакт.

Если $F$ в-пн. сн. в точке $t_{0}$, то оно пн. сн. в точке $t_{0}$. Обратное верно, если $F\left(t_{0}\right)$ - компакт. 
ДокАЗАТЕЛЬСтво. Утверждение о связи пн. св. с $\varepsilon$-пн. св. доказано в замечании главы $3 \S 1$ из [1].

Пусть $y_{0} \in F\left(t_{0}\right)$ и $N\left(y_{0}\right)$ - окрестность $y_{0}$. Найдется окрестность точки $t_{0} U\left(t_{0}\right)$ такая, что

$$
\forall t \in U\left(t_{0}\right) \quad y_{0} \in F\left(t_{0}\right) \subset F(t)+B_{\varepsilon}(0)
$$

и $B_{\varepsilon}\left(y_{0}\right) \subset N\left(y_{0}\right)$. Из этих условий получаем, что для всех $t \in U\left(t_{0}\right)$ вьполняется условие

$$
F(t) \cap N\left(y_{0}\right) \supset F(t) \cap B_{\varepsilon}\left(y_{0}\right) \neq \varnothing .
$$

Пусть $F$ пн. сн. в точке $t_{0}$. Допустим, что

$$
\exists \varepsilon>0 \exists t_{k} \rightarrow t_{0} \exists y_{k} \in F\left(t_{0}\right) \quad \varrho\left(y_{k}, F\left(t_{k}\right)\right) \geqslant \varepsilon .
$$

Так как $F\left(t_{0}\right)$ - компакт, то без ограничения общности $y_{k} \rightarrow y_{0} \in F\left(t_{0}\right)$. В силу определения пн. сн. существует $k_{0}$ такое, что для всех $k>k_{0}$ расстояние $\varrho\left(y_{0}, F\left(t_{k}\right)\right) \leqslant \varepsilon / 2$. Имеем

$$
\varepsilon \leqslant \varrho\left(y_{k}, F\left(t_{k}\right)\right) \leqslant \varrho\left(y_{k}, y_{0}\right)+\varrho\left(y_{0}, F\left(t_{k}\right)\right),
$$

что противоречит (2.1) при больших $k$.

Графиком многозначного отображения $F: \mathscr{T} \rightarrow \mathscr{Y}$ называется множество

$$
\operatorname{gf} F=\{(t, x) \in \mathscr{T} \times \mathscr{Y} \mid t \in \mathscr{T}, \quad x \in F(t)\}
$$

ЛЕмма $2.2[1$, гл. $3, \S 1$, следствие 9$]$. Пусть многозначное отображсение $F: \mathscr{T} \rightarrow \mathscr{Y}$ имеет замкнутый график и пространство У компактно. Тогда $F$ полунепрерывно сверху.

ТЕОрема 2.1. Пусть $\mathscr{T ~ - ~ п о л н о е ~ м е т р и ч е с к о е ~ п р о с т р а н с т в о , ~ E ~ - ~ с е п а р а б е л ь - ~}$ ное банахово пространство. Пусть $F, G: \mathscr{T} \rightarrow E$ - многозначные отображсения такие, что образы $F$ и $G$ слабо компактны в $E, F$ слабо пн. св. в $E, G$ слабо пн. cн. в $E$. Пусть для всех $t \in \mathscr{T}$ множсество $H(t)=F(t) \stackrel{*}{*} G(t) \neq \varnothing$ и образ $H(\mathscr{T})$ содержится в слабо компактном множестве. Тогда отображсние $H$ пн. св. на $\mathscr{T}$ в слабой топологии $E$.

ДокАЗАТЕЛЬСТво. Пусть $\tau$ - топология в $\mathscr{T}, \tau_{w}$ - слабая топология в $E$.

Поскольку $E$ сепарабельно, то в силу теоремы 3 главы V 66 из [4] пространство $E$ можно снабдить инвариантной относительно сдвигов метрикой $\varrho$ такой, что топология $\tau_{1}$, индуцированная этой метрикой, слабее топологии $\tau_{w}$ и совпадает с топологией $\tau_{w}$ на слабых компактах из $E$.

Пусть $B_{\varepsilon}(0)=\{x \in E \mid \varrho(0, x) \leqslant \varepsilon\}$.

Покажем, что график отображения $H$ замкнут в пространстве $\left(\mathscr{T} \times E, \tau \times \tau_{1}\right)$.

Пусть $\left(t_{k}, x_{k}\right) \in \operatorname{gf} H ; t_{k} \rightarrow t_{0} \in \mathscr{T}, x_{k} \rightarrow x_{0} \in E$.

Из полунепрерьвности $F$ и $G$ в слабой топологии и того, что $\tau_{1}$ слабее $\tau_{w}$, следует, что $F$ и $G$ пн. в топологии $\tau_{1}$.

Из $\tau_{w}$-компактности значений $F$ и $G$ следует их $\tau_{1}$-компактность.

По лемме 2.1 отображение $F \varepsilon$-пн. св., а отображение $G \varepsilon$-пн. сн. в $\tau_{1}$ топологии пространства $E$. 
Фиксируем $\varepsilon>0$. Имеем

$$
\begin{gathered}
x_{k}+G\left(t_{k}\right) \subset F\left(t_{k}\right) \forall k, \quad G\left(t_{0}\right) \subset G\left(t_{k}\right)+B_{\varepsilon}(0) \forall k \geqslant k_{2}(\varepsilon), \\
x_{0} \in x_{k}+B_{\varepsilon}(0) \forall k \geqslant k_{1}(\varepsilon), \quad F\left(t_{k}\right) \subset F\left(t_{0}\right)+B_{\varepsilon}(0) \forall k \geqslant k_{3}(\varepsilon) .
\end{gathered}
$$

Взяв $k>\max _{1 \leqslant i \leqslant 3} k_{i}(\varepsilon)$, получаем, что

$$
x_{0}+G\left(t_{0}\right) \subset F\left(t_{0}\right)+\sum_{i=1}^{3} B_{\varepsilon}(0) .
$$

Так как последнее включение имеет место для любых $\varepsilon>0$, a $\left\{B_{\varepsilon}(0)\right\}_{\varepsilon>0}-$ локальная база нуля в $\left(E, \tau_{1}\right)$ и $F\left(t_{0}\right) \tau_{1}$-замкнуто, то $x_{0} \in H\left(t_{0}\right)$. Мы получили, что gf $H$ замкнут в $\left(\mathscr{T} \times E, \tau \times \tau_{1}\right)$.

Так как $H(\mathscr{T})$ содержится по условию в слабом компакте, а топологии $\tau_{1}$ и $\tau_{w}$ на этом слабом компакте совпадают, то график gf $H$ замкнут в $\left(\mathscr{T} \times E, \tau \times \tau_{w}\right)$. Отсюда по лемме 2.2 получаем, что $H$ слабо полунепрерьвно сверху в любой точке $t \in \mathscr{T}$.

3. Непрерывность разности многозначных отображений в $\mathbb{R}^{n}$. Напомним, что выпуклая функция на $\mathbb{R}^{n}$ непрерьвна на любом относительно открытом подмножестве эффективного множества функции. Однако выпуклая полунепрерывная снизу функция может не быть полунепрерьвной сверху на относительной границе своей области определения (см. [5, гл. II, §10]).

Условимся назьвать множество $S \subset \mathbb{R}^{n}$ локально симплициальньм, если для всех $x \in S$ найдется конечное число симплексов $S_{1}, \ldots, S_{m}$, содержащихся в $S$ и таких, что для некоторой окрестности $U$ точки $x$ имеет место равенство

$$
U \cap\left(\bigcup_{i=1}^{m} S_{i}\right)=U \cap S .
$$

Лемма 3.1 [5, гл. II, $\S 10$, теорема 10.2]. Пусть $f-$ выпуклая функиия в $\mathbb{R}^{n}$ и $S-$ произвольное локально симплициальное подмножсество из әффективного множества $f$. Тогда $f$ полунепрерывна сверху на $S$.

ТеОрема 3.1. Пусть выпуклый компакт $F$ имеет вид $F=P \cap Q$, дде $P \subset \mathbb{R}^{n}-$ многогранник, а $Q \subset \mathbb{R}^{n}$ - строго выпуклый компакт (в частности, $F$ может быть многогранником). Пусть $G(t)$ - непрерывное (в метрике Хаусдорфа) многозначное отображсене с компактными значениями такое, что $H(t)=F \stackrel{*}{G(t)}$ $\neq \varnothing$. Тогда многозначное отображение $H(t)$ непрерывно.

ДокАЗАТЕЛьСтво. Полунепрерьвность сверху $H(t)$ следует из теоремы 2.1. Докажем полунепрерывность $H(t)$ снизу (пн. сн.).

Допустим, в точке $t_{0}$ условие пн. сн. нарушается, т.е.

$$
\exists \varepsilon_{0}>0, \exists x \in H\left(t_{0}\right), \exists t_{k} \rightarrow t_{0}: \quad x \notin H\left(t_{k}\right)+B_{\varepsilon_{0}}(0) .
$$

Пусть $x_{k} \in H\left(t_{k}\right)$. Так как

$$
\left\{x_{k}\right\}_{k=1}^{\infty} \subset F-\bigcup_{k=1}^{\infty} G\left(t_{k}\right),
$$


то в силу ограниченности последовательности $x_{k}$ можно без ограничения общности считать, что $x_{k} \rightarrow x_{0} \neq x$. Поскольку $H(t)$ пн. св., то $x_{0} \in H\left(t_{0}\right)$. Далее будем без ограничения общности считать, что $x_{0}=0$. Пусть $G_{1}\left(t_{k}\right)=G\left(t_{k}\right)+x_{k}, H_{1}\left(t_{k}\right)=F \stackrel{*}{*} G_{1}\left(t_{k}\right)$, $0 \leqslant k \leqslant \infty$.

Из условия (3.1) и сходимости $x_{k} \rightarrow 0$ получаем, что, начиная с некоторого $K_{0}$, для всех $k>K_{0}, k \neq 0$, вьполнено

$$
x \notin H_{1}\left(t_{k}\right)+B_{\varepsilon_{0} / 2}(0) .
$$

Отметим, что $x \neq 0$.

Разложим $\mathbb{R}^{n}$ в прямую сумму: $\mathbb{R}^{n}=L \oplus \operatorname{lin}[x]$. Здесь $L$ есть $(n-1)$-мерное подпространство. Без ограничения общности можно считать вектор $-x$ сонаправленным с $n$-й декартовой координатной осью $O x_{n}$. Пусть $\pi$ есть ортогональньй проектор на $L$.

Введем вьпуклую функцию $f$, действующую из $\pi F \subset L$ в $O x_{n}$ и задаваемую формулой $f(w)=\min \{\mu:(w, \mu) \in F\}$. Легко видеть, что $f(w)=\max \left\{f_{P}(w), f_{Q}(w)\right\}$, где $f_{P}: \pi P \rightarrow O x_{n}, f_{P}(w)=\min \{\mu:(w, \mu) \in P\}$, a $f_{Q}: \pi Q \rightarrow O x_{n}, f_{Q}(w)=\min \{\mu:$ $(w, \mu) \in Q\}$.

Покажем, что функции $f_{P}$ и $f_{Q}$ непрерьвны на $\pi P$ и $\pi Q$ соответственно.

Полунепрерывность снизу функций $f_{P}$ и $f_{Q}$ непосредственно следует из замкнутости лебеговых множеств функций $f_{P}$ и $f_{Q}[5$, гл. II, $\S 7$, теорема 7.1].

Поскольку $\pi P$ есть многогранник и, следовательно, локально симплициальное множество (см. [5, гл. II, §10]), то по лемме 3.1 функция $f_{P}$ полунепрерывна сверху на множестве $\pi P$.

Покажем полунепрерьвность сверху функции $f_{Q}$ на множестве $\pi Q$. Достаточно проверить пн. св. на относительной границе множества $\pi Q$. От противного: пусть существуют $w \in \partial_{\mathrm{ri}} \pi Q, \delta>0$ и $w_{k} \rightarrow w, w_{k} \in \pi Q$, такие, что $\lim _{k \rightarrow \infty} f_{Q}\left(w_{k}\right) \geqslant f_{Q}(w)+\delta$. Поскольку точки $\left(w_{k}, f_{Q}\left(w_{k}\right)\right)$ лежат в компакте $Q$, то $\left(w_{k}, f_{Q}\left(w_{k}\right)\right) \rightarrow(w, f) \in Q$, причем $f \geqslant f_{Q}(w)+\delta$. Так как прообраз относительно граничных точек $\partial_{\text {ri }} \pi Q$ есть граничные точки $Q$, то верно включение $\left[\left(w, f_{Q}(w)\right),(w, f)\right] \subset \partial Q$, что противоречит строгой выпуклости $Q$. Отсюда $f_{Q}$ полунепрерывна сверху на $\pi Q$.

Из непрерывности $f_{P}$ и $f_{Q}$ на $\pi P$ и $\pi Q$ вытекает их непрерывность на $\pi F \subset \pi P \cap \pi Q$. Отсюда следует, что функция $f$ непрерьвна на $\pi F$ и, следовательно, равномерно непрерьвна.

В силу полунепрерьвности сверху отображения $G(t)$ в точке $t_{0}$ существует бесконечно малая последовательность $\left\{\alpha_{k}\right\}$ такая, что

$$
G_{1}\left(t_{k}\right) \subset G_{1}\left(t_{0}\right)+\alpha_{k} B_{1}(0)
$$

Запишем условие равномерной непрерывности $f$ на $\pi F$ в следующем виде:

$$
\forall m \exists k_{m}:\left|f\left(w_{1}\right)-f\left(w_{2}\right)\right| \leqslant \frac{1}{m} \quad \forall k \geqslant k_{m} \forall w_{1}, w_{2} \in \pi F:\left|w_{1}-w_{2}\right| \leqslant \alpha_{k} .
$$

Пусть $m$ и $k=k_{m}$ выбраны так, что $k>K_{0}$ и $2 \alpha_{k}+1 / m<(1 / 2) \min \left\{\varepsilon_{0},|x|\right\}$.

Фиксируем произвольную точку $u_{k} \in G_{1}\left(t_{k}\right)$.

1. Если $x+u_{k} \in F$, то из включения $u_{k} \in F$ и выпуклости $F$ следует, что $u_{k}+\lambda x \in F$ для всех $\lambda \in[0,1]$. 
2. Пусть $x+u_{k} \notin F$. Из условия (3.3) найдется $u_{0}^{k} \in G_{1}\left(t_{0}\right):\left|u_{0}^{k}-u_{k}\right| \leqslant \alpha_{k}$. Отметим, что $u_{0}^{k}+x \in F$. Пусть $w_{k}=\pi u_{k}, w_{0}^{k}=\pi u_{0}^{k} ;\left|w_{k}-w_{0}^{k}\right| \leqslant \alpha_{k}$. Определим вектор $z_{k}=\left(w_{k}, f\left(w_{k}\right)\right)-u_{k}-x$.

Будем говорить, что точка $(w, \mu)$ лежит выше $(w, \lambda)$, если $\mu \geqslant \lambda$.

Рассмотрим точки $x+u_{k},\left(w_{k}, f\left(w_{k}\right)\right), u_{0}^{k}+x$ и $\left(w_{0}^{k}, f\left(w_{0}^{k}\right)\right)$. Они лежат на параллельных прямых aff $\left\{u_{k}, u_{k}+x\right\}$ и aff $\left\{u_{0}^{k}, u_{0}^{k}+x\right\}$, причем точка $\left(w_{k}, f\left(w_{k}\right)\right)$ лежит вьше $u_{k}+x$, a $u_{0}^{k}+x$ выше $\left(w_{0}^{k}, f\left(w_{0}^{k}\right)\right)$, т.е. отрезки $\left[x+u_{k}, x+u_{0}^{k}\right]$ и $\left[\left(w_{k}, f\left(w_{k}\right)\right),\left(w_{0}^{k}, f\left(w_{0}^{k}\right)\right)\right]$ пересекаются в точке $a$ как диагонали трапеции с вершинами в точках $x+u_{k},\left(w_{k}\right.$, $\left.f\left(w_{k}\right)\right), u_{0}^{k}+x$ и $\left(w_{0}^{k}, f\left(w_{0}^{k}\right)\right)$. Тогда

$$
\begin{aligned}
\left|z_{k}\right| & \leqslant\left|\left(w_{k}, f\left(w_{k}\right)\right)-a\right|+\left|a-u_{k}-x\right| \\
& \leqslant\left|w_{k}-w_{0}^{k}\right|+\left|f\left(w_{k}\right)-f\left(w_{0}^{k}\right)\right|+\left|u_{k}-u_{0}^{k}\right| .
\end{aligned}
$$

Отметим, что если прямые aff $\left\{u_{k}, u_{k}+x\right\}$ и aff $\left\{u_{0}^{k}, u_{0}^{k}+x\right\}$ совпадают, то $w_{k}=w_{0}^{k}$ и полученная выше оценка очевидна. Первое и третье слагаемые в правой части предыдущей формулы не превосходят $\alpha_{k}$. В силу равномерной непрерывности $f$ на компакте $\pi F$ выполнена оценка $\left|f\left(w_{k}\right)-f\left(w_{0}^{k}\right)\right| \leqslant 1 / m$.

Из пунктов 1 и 2 получаем, что для любого $u_{k} \in G_{1}\left(t_{k}\right)$

$$
u_{k}+x-\left(\frac{1}{m}+2 \alpha_{k}\right) \frac{x}{|x|} \in F,
$$

откуда

$$
x-\left(\frac{1}{m}+2 \alpha_{k}\right) \frac{x}{|x|} \in H_{1}\left(t_{k}\right),
$$

т.е.

$$
x \in H_{1}\left(t_{k}\right)+B_{\varepsilon_{0} / 2}(0),
$$

что противоречит (3.2). Итак, $H(t)$ пн. сн.

СЛЕДСТВИЕ 3.1. Для любого выпуклого компакта $F \subset \mathbb{R}^{2}$ и для любого непрерывного многозначного отображсения $G: \mathscr{T} \rightarrow \mathbb{R}^{2}$ такого, что $H(t)=F \stackrel{*}{*} G(t) \neq \varnothing$, отобрахсение $H(t)$ непрерывно.

Для доказательства заметим, что любое выпуклое множество на оси локально симплициально, откуда следует полунепрерьвность сверху функции $f$ (в обозначениях теоремы 3.1). В остальном доказательство повторяет доказательство теоремы 3.1.

ПримеР 3.1. Покажем, что условия теоремы 3.1 относительно вида $F$ существенны. Рассмотрим $\mathbb{R}^{3}$ со стандартной декартовой системой координат. Через со будем обозначать оператор взятия вьпуклой оболочки.

Пусть $a_{1}=(1,0,0), a_{2}=(-1,0,0), a_{3}=(1,0,1), a_{4}=(-1,0,1)$. Рассмотрим также две дуги окружности радиуса 1 радианной меры $\pi$ :

$$
\begin{array}{ll}
D_{1}=\left\{x_{3}=0, x_{1}=\cos t, x_{2}=\sin t,\right. & t \in[0, \pi]\} \\
D_{2}=\left\{x_{3}=1, x_{1}=\cos t, x_{2}=\sin t,\right. & t \in[\pi, 2 \pi]\} .
\end{array}
$$

Пусть $F_{1}=\operatorname{co}\left\{\left\{a_{3}, a_{4}\right\} \cup D_{1}\right\}, F_{2}=\operatorname{co}\left\{\left\{a_{1}, a_{2}\right\} \cup D_{2}\right\}$. 
$F=F_{1} \cup F_{2}$. Легко видеть, что $F$ выпукло и не является пересечением многогранника и строго выпуклого компакта.

Пусть $a(t)=(\cos t, \sin t, 0), G(t)=\operatorname{co}\left\{a_{1}, a_{2}, a(t)\right\}$.

Пусть $t \in[\pi / 2,3 \pi / 2]$. Имеем

$$
F * G(t)= \begin{cases}\{(0,0,0)\}, & t \in[\pi / 2, \pi) \\ \operatorname{co}\{(0,0,0),(0,0,1)\}, & t=\pi \\ \{(0,0,1)\}, & t \in(\pi, 3 \pi / 2] .\end{cases}
$$

Итак, отображение $F \stackrel{*}{*} G(t), t \in[\pi / 2,3 \pi / 2]$, не только не непрерьвно, но даже не имеет непрерьвного селектора.

ТЕОРема 3.2. Пусть выпуклый компакт $F$ имеет вид $F=P \cap Q$, дде $P \subset \mathbb{R}^{n}$ многогранник, а $Q \subset \mathbb{R}^{n}$ - строго выпуклый компакт, и пусть матрица $T(t)$ размера $n \times n$ с непрерывными компонентами невырожсдена для всех $t \in \mathscr{T}$. Пусть $G: \mathscr{T} \rightarrow \mathbb{R}^{n}$ непрерывное многозначное отображсение и для всех $t \in \mathscr{T} H(t)=$ $(T(t) F) \stackrel{*}{*} G(t) \neq 0$. Тогда многозначное отображсение $H$ непрерывно на $\mathscr{T}$.

ДокАЗАТЕЛЬСТВо. Пусть $G_{1}(t)=T^{-1}(t) G(t), H_{1}(t)=T^{-1}(t) H(t)$.

Так как $H_{1}(t)+G_{1}(t) \subset F$ для всех $t \in \mathscr{T}$, то $H_{1}(t) \subset F \stackrel{*}{*} G_{1}(t)$.

Пусть $x \in F \stackrel{*}{*} G_{1}(t)$. Тогда $T(t) x+G(t) \subset T(t) F$, т.е. $T(t) x \in H(t)$, откуда $x \in H_{1}(t)$.

Итак, $H_{1}(t)=F * G_{1}(t)$, по теореме $3.1 H_{1}(t)$ непрерьвно, отсюда $H(t)$ непрерывно.

ПРИмеР 3.2. Отметим, что в теореме 3.2 нельзя отказаться от обратимости $T(t)$. Пусть $F=\operatorname{co}\{(0,0,0),(0,0,2),(1,0,0),(1,0,1),(0,1,0),(0,1,2),(1,1,0),(1,1,1)\}, T(t)$, $t \in[0, \pi / 4]$, есть суперпозиция поворота на угол $t$ вокруг оси $O x_{1}$ (направление поворота от оси $O x_{2}$ к $\left.O x_{3}\right)$ и ортогонального проектирования на $O x_{1} x_{2}$. Пусть

$$
G(t)=\operatorname{co}\{(0,-2 \sin t, 0),(0, \cos t, 0)\} .
$$

Тогда

$$
T(t) F=\operatorname{co}\{(0, \cos t, 0),(1, \cos t, 0),(1,-\sin t, 0),(0,-2 \sin t, 0)\},
$$

поэтому отображение $(T(t) F) \stackrel{*}{G} G(t)$ не пн. сн. в нуле:

$$
(T(t) F) * G(t)= \begin{cases}\{(0,0,0)\}, & t \in(0 ; \pi / 4], \\ \operatorname{co}\{(0,0,0),(1,0,0)\}, & t=0 .\end{cases}
$$

\section{СПИСОК ЦИТИРОВАННОЙ ЛИТЕРАТУРЫ}

[1] Обен Ж.-П., Экланд И. Прикладной нелинейный анализ. М.: Мир, 1988.

[2] Половинкин Е.С. Элементы теории многозначных отображений. Учеб. пособие. М.: МФТИ, 1982.

[3] Понтрягин Л.С. Линейные дифференциальные игры преследования // Матем. сб. 1980. Т. 112. № 3. C. $307-330$.

[4] Данфорд Н., Шварц Дж. Линейные операторы. Общая теория. М.: ИЛ, 1962.

[5] Рокафеллар Р. Вьпукльй анализ. М.: Мир, 1973. 